\title{
Avaliação do perfil motor em crianças de Teresina - PI com Transtorno do Espectro Autista (TEA)
}

\author{
Evaluation of the motor profile in children of Teresina - PI with Autistic \\ Spectrum Disorder (ASD)
}

Evaluación del perfil motor en niños de Teresina - PI con Transtorno del Espectro Autista (TEA)

\author{
* Bruna Marques Teixeira \\ Graduanda pela Universidade Estadual do Piauí (UESPI), Teresina, Piauí, Brasil. \\ bruna_marques.mt@hotmail.com - https://orcid.org/0000-0001-7410-2825

\section{** Fabiana Teixeira de Carvalho} \\ Professora Doutora Adjunta da Universidade Estadual do Piauí (UESPI), Teresina, Piauí, Brasil. \\ fabianatcarvalho@hotmail.com
}

\section{*** Jaqueline Raíssa Lopes Vieira}

Graduanda pela Universidade Estadual do Piauí (UESPI), Teresina, Piauí,

Brasil.jaque.castelamary@gmail.com

Recebido em 13 de julho de 2018

Aprovado em 20 de maio de 2019

Publicado em 00 de julho de 2019

\section{RESUMO}

Uma das principais comorbidades associadas ao autismo em crianças relaciona-se diretamente ao déficit do desempenho motor como resposta ao movimento. Crianças com transtorno do espectro autista (TEA) apresentam uma série de alterações motoras, com atrasos na coordenação, resultando em déficits na aprendizagem de habilidades motoras finas e complexas. O objetivo do estudo foi avaliar o perfil motor de crianças com transtorno do espectro autista (TEA). Trata-se de um estudo clínico, observacional, transversal, prospectivo, quantitativo e descritivo. A pesquisa foi realizada com crianças com diagnóstico de TEA, de ambos os sexos, com idades entre 05 e 11 anos, em uma Associação de crianças autistas em Teresina-PI. Para a realização do estudo utilizou-se a Escala de Desenvolvimento Motor (EDM), que compreende provas diversificadas e de diferentes dificuldades que permitem avaliar o nível de desenvolvimento motor. Os resultados revelaram diferença significativa entre a idade motora e a idade cronológica, com nível de desenvolvimento motor inferior e muito inferior. Foi possível observar que crianças com TEA apresentam idade motora geral inferior à idade cronológica.

Palavras-chave: Desenvolvimento motor; Transtorno do espectro autista; Crianças. 


\section{ABSTRACT}

One of the major comorbidities associated with autism in children is directly related to motor performance deficit as a response to movement. Children with Autism Spectrum Disorder (ASD) have a number of motor changes, with delays in coordination, resulting in deficits in learning fine and complex motor skills. The objective of the study was to evaluate the motor profile of children with ASD. It is a clinical, observational, transversal, prospective, quantitative and descriptive study. The study was carried out with 20 children diagnosed with ASD, of both sexes, aged between 05 and 11 years, in an Association of autistic children in Teresina-PI. The Motor Development Scale (EDM) was used to carry out the study, which includes diverse tests and different difficulties that allow to evaluate the level of motor development. The results revealed a significant difference between motor age and chronological age, with a lower and lower motor development level. It was possible to observe that children with ASD present general motor age inferior to chronological age.

Keywords: Motor development; Autism spectrum disorder; Children.

\section{RESUMEN}

Una de las principales comorbilidades asociadas al autismo en niños se relaciona directamente al déficit del desempeño motor como respuesta al movimiento. Los niños con trastorno del espectro del autismo (TEA) presentan una serie de cambios motor, con retrasos en la coordinación, resultando en déficit en el aprendizaje de habilidades motoras finas y complejas El objetivo del estudio fue evaluar el perfil motor de niños con TEA. Se trata de un estudio clínico, observacional, transversal, prospectivo, cuantitativo y descriptivo. La investigación fue realizada con 20 niños con diagnóstico de TEA, de ambos sexos, con edades entre 05 y 11 años, en una Asociación de niños autistas en Teresina-PI. Los niños con trastorno del espectro del autismo (TEA) presentan una serie de cambios motor, con retrasos en la coordinación, resultando en déficit en el aprendizaje de habilidades motoras finas y complejas. El objetivo del estudio fue evaluar el perfil motor de niños con TEA.Para la realización del estudio se utilizó la Escala de Desarrollo Motor (EDM), que comprende pruebas diversificadas y de diferentes dificultades que permiten evaluar el nivel de desarrollo motor. Los resultados revelaron una diferencia significativa entre la edad motora y la edad cronológica, con un nivel de desarrollo motor inferior y muy inferior. Es posible observar que los niños con TEA presentan una edad motora general inferior a la edad cronológica.

Palabras clave: Desarrollo motor; Trastorno del espectro autista; Los niños.

\section{Introdução}

O termo autismo passou por uma série de modificações no decorrer dos anos até ser referido como Transtorno do Espectro Autista (TEA) pelo Manual Diagnóstico e Estatístico de Transtornos Mentais (DSM-5), que o definiu como distúrbio do desenvolvimento, marcado por atrasos na comunicação e na interação social, com padrões de atividades 
repetidas e restritas, associados à diminuição ou perda dessas habilidades, apresentando pelo menos duas das seguintes manifestações: movimentos ou fala estereotipadas; insistência em padrões de comportamento verbal ou não verbal; interesses excessivos e anormais no desempenho de determinadas tarefas; aumento ou diminuição da resposta a estímulos sensoriais (AMERICAN PSYCHIATRIC ASSOCIATION, 2014).

Em crianças com TEA pode-se observar variação no tônus, bem como hipotonia moderada em mais de 50\% dos casos (SACREY et al., 2014). O mesmo estudo afirma que, além de prejuízos na comunicação, alguns distúrbios podem estar presentes, como padrões motores anormais, incluindo alterações nas habilidades motoras finas e grossas, dificuldades na execução de movimentos precisos e atrasos na aprendizagem motora.

Ainda segundo o DSM-5 (APA, 2014), para que se estabeleça um diagnóstico, devese confirmar o atraso na comunicação social em comparação ao nível geral do desenvolvimento. Para Onzi e Gomes (2015), o TEA apresenta uma alta complexidade, com uma definição distante de abranger com exatidão toda a sua extensão, considerando que as pesquisas realizadas atualmente apontam para uma limitação de meios para a sua identificação.

Entender as etapas, bem como identificar atrasos ou desvios do desenvolvimento exige um conhecimento prévio das fases que cursam com o seu processo natural, além da variabilidade existente em cada indivíduo. O neurodesenvolvimento é um processo dinâmico, que se processa a partir de uma rede integrativa que envolve motricidade, linguagem, autonomia, adaptações. Contudo, identificar atrasos leves nos primeiros anos de vida não tem mostrado ser fácil, demonstrando a dificuldade em estabelecer o diagnóstico precoce (OLIVEIRA, 2009). O diagnóstico se baseia em atrasos na interação social e na comunicação, com padrões restritos e estereotipados do comportamento, sendo considerado um diagnóstico complexo, com dificuldades para a confirmação do transtorno, uma vez que a grande maioria dos pacientes apresenta os sintomas nos primeiros anos de vida (CANUT et al., 2014; OLIVEIRA, 2010; NIKOLOV; JONKER; SCAHILL, 2006).

O TEA não apresenta uma etiologia específica, acreditando-se estar associada a fatores genéticos, ambientais, imunológicos e neurológicos, desencadeando alterações que contribuem para o atraso no desenvolvimento motor (CANUT et al., 2014). desenvolvimento motor é definido como um processo contínuo, associado à idade cronológica e de interações com o meio, possibilitando à criança adquirir habilidades que 
variam desde movimentos simples a complexos (ROSA NETO et al., 2010; WILLRICH; AZEVEDO; FERNANDES, 2009).

Considerando indivíduos com TEA, há evidências da idade motora geral apresentando-se menor que a esperada para a idade cronológica (OKUDA; MISQUIATTI; CAPELLINI, 2010). MacNeil e Mostofsky (2012) avaliaram três grupos de crianças com TEA, desenvolvimento típico e Transtorno de Déficit de Atenção (TDAH) quanto ao desenvolvimento. Foi possível observar que o grupo com TEA obteve prejuízos em seu desempenho motor quando comparado aos demais. Gonzaga et al., 2015 verificaram em seu estudo com a participação de seis crianças com TEA, com a média de idade de 57,50 $\pm 17,12$ meses, que $83,34 \%$ dessas apresentaram déficits no desenvolvimento da psicomotricidade. O mesmo foi identificado no estudo de Okuda et al. (2010), demonstrando que as 6 crianças avaliadas com TEA apresentaram idade motora geral inferior à idade cronológica ao avaliar motricidade fina, motricidade global, equilíbrio, esquema corporal, organização espacial, organização temporal e lateralidade.

Para que determinada função seja desenvolvida precisa-se de um amadurecimento neuronal anatômico, decorrente de uma sucessão de fases que vai desde a proliferação e migração neuronal até a mielinização. Acredita-se que a produção elevada de mielina associada à eliminação neuronal, axonal e de sinapses, nas primeiras fases de vida seja de grande importância no desenvolvimento neuropsicomotor (DNPM), em geral, com evidências de que a mielinização elevada pode justificar alterações neuropsicomotoras no último trimestre de vida (NITRINI; BACHESCHI, 2008).

Disfunções motoras podem surgir durante o desenvolvimento motor da criança, como o Transtorno do Desenvolvimento da Coordenação (TDC), o que dificulta o diagnóstico e tratamento especializado (PULZI; RODRIGUES, 2015). Os sinais estão geralmente associados a realização de tarefas de forma inconsistente, com coordenação motora deficitária, ritmo diminuido, aumento da tensão corporal e maior recrutação muscular nas atividades, apresentando dificuldades na execução de tarefas simples, como se vestir, em atividades de lazer e em atividades escolares, somadas aos problemas de interação social (MAGALHÃES; NASCIMENTO; REZENDE, 2004).

Uma das principais comorbidades associadas ao autismo em crianças relaciona-se diretamente ao déficit do desempenho motor como resposta ao movimento (MOSTOFSKY et al., 2006). Crianças com TEA apresentam uma série de alterações motoras, com atrasos 
na coordenação, resultando em déficits na aprendizagem de habilidades motoras finas e complexas, que incluem motricidade global, equilíbrio, esquema corporal, e organização espacial e temporal (LARSON et al., 2008). No estudo de Okuda et al. (2010), cujas crianças com TEA foram avaliadas quanto a motricidade fina e global, equilíbrio, esquema corporal e espacial e lateralidade, o desempenho motor apresentado foi inferior ao desenvolvimento esperado para a idade, confirmando os atrasos Transtornos do Espectro do Autismo (TEA). O mesmo foi observado por Anjos et al. (2017) em sua pesquisa realizada com 30 crianças diagnosticadas com transtorno do espectro autista, concluindo que as crianças avaliadas estavam com atrasos motores significativos.

A descoberta de casos de autismo tem crescido nos últimos anos no Brasil e em todo o mundo (ONZI; GOMES, 2015). No país, a estimativa era de cerca de 500 mil pessoas com autismo, em 2010; a nível mundial estima-se que o sexo masculino seja o mais acometido e que um a cada 88 nascidos vivos apresente TEA (GOMES et al., 2015). Embora a identificação e o diagnóstico precoce do autismo no país tenham aumentado consideravelmente, a ausência e a dificuldade em estabelecer o diagnóstico ainda configura-se como uma realidade (SILVA; MULICK, 2009).

Evidências científicas mostram o importante papel do fisioterapeuta no desenvolvimento de indivíduos com TEA, como verificado por Okuda et al. (2010), relatando que após intervenção psicomotora houve melhora de $100 \%$ das crianças avaliadas no desempenho motor. Entretanto, a relação da fisioterapia e seus métodos de tratamento são pouco difundidos entre os profissionais da área, o que dificulta na elaboração e na realização de condutas necessárias para a evolução desses pacientes (SEGURA; NASCIMENTO; KLEIN, 2011).

Entre as principais causas de prejuízos no desenvolvimento estão os transtornos do espectro autista, apresentando como consequência impactos socioeconômicos e sobrecarga emocional para a família (NIKOLOV; JONKER; SCAHILL, 2006). Além disso, o crescente número de casos de TEA, associado ao elevado custo de tratamento, apontam para a necessidade de novos métodos de intervenção. Dados do Ministério da saúde mostram que foi instituída a Política Nacional de Proteção dos Direitos da Pessoa com Transtornos do Espectro do Autismo, considerando a pessoa autista como deficiente legal. Salientando a necessidade de assistência à criança e considerando que a ocorrência dessa condição pode gerar limitações nas capacidades motoras e sociais, demandando cuidados 
que reforcem a reabilitação, a oferta de tratamento nas redes de cuidados a pessoa com deficiência configura-se como estratégia de intervenção (BRASIL, 2014).

Dessa forma, a avaliação do perfil motor permite identificar possíveis atrasos no desenvolvimento, contribuindo para o diagnóstico e intervenções precoces (MIERES, et al., 2012; SANDRONI; CIASCA; RODRIGUES, 2015). Assim, o presente estudo teve como objetivo avaliar o perfil motor de crianças com TEA.

\section{Metodologia}

\section{Participantes}

Trata-se de um estudo clínico, observacional, transversal, prospectivo, quantitativo e descritivo, realizado em uma associação de crianças autistas. A amostra constituiu-se de 20 crianças com diagnóstico de Transtorno do Espectro Autista (TEA), com idades de 5 a 11 anos, de ambos os gêneros, em Teresina - PI. A média e desvio padrão da Idade Cronológica apresentada foi, respectivamente, 106,75 e 20,55 meses. A média da ldade Motora Geral, 57,4 meses, e Desvio Padrão, 11,93 meses. O grau de escolaridade variou do segundo período do ensino infantil ao sétimo ano do ensino fundamental.

\section{Cuidados éticos}

O estudo foi aprovado pelo Comitê de Ética em Pesquisa da Universidade Estadual do Piauí, sob protocolo de número 2.537.168.

\section{Instrumentos}

Para a realização do estudo, utilizou-se a Escala de Desenvolvimento Motor (EDM) de Rosa Neto (2015), que identifica alterações do desenvolvimento infantil, bem como algumas perturbações na coordenação motora. A escala avaliativa compreende provas diversificadas e de diferentes dificuldades que permitem avaliar o nível de desenvolvimento motor, ou seja, a idade motora da criança em cada uma das variáveis: motricidade fina (IM1), motricidade global (IM2), equilíbrio (IM3), esquema corporal (IM4), organização espacial (IM5), organização temporal (IM6) e lateralidade, sendo realizados de acordo com os critérios estabelecidos no manual de avaliação motora.

A Idade Motora (IM) é referida como a idade que está associada ao sucesso da realização da prova pela criança, podendo-se determinar um avanço ou um atraso motor em determinada habilidade pela comparação com a idade cronológica (IC). O Quociente Motor (QM) é obtido com base na divisão da idade motora de determinada habilidade pela 
idade cronológica da criança, multiplicando o resultado por 100. A idade negativa ou positiva (IN/IP) é obtida pela diferença entre a idade motora geral e a idade cronológica, com base na obtenção de valores numéricos superiores da idade motora geral em relação à idade cronológica, expressa geralmente em meses (GONZAGA et al., 2015).

\section{Procedimentos de coleta}

Os critérios de inclusão foram: possuir diagnóstico médico para o TEA, receber atendimento na Associação de crianças autistas e possuir idade entre 02 e 11 anos. Foram excluídas da pesquisa as crianças que por algum motivo não conseguiram finalizar a avaliação, que apresentaram outros distúrbios associados, como TDAH, paralisia cerebral, hiperatividade, e que, por motivos próprios, os responsáveis retiraram o seu Termo de Consentimento Livre e Esclarecido.

As avaliações foram realizadas após a assinatura do Termo de Consentimento Livre e Esclarecido pelos pais e/ou responsáveis e do Termo de Assentimento pelos participantes, em que foram explicados os procedimentos da avaliação, bem como os seus objetivos.

Os dados foram coletados no período de abril a maio de 2018, em duas etapas: a primeira, referente a coleta dos dados do prontuário da criança, em que se verificou 0 diagnóstico clínico de TEA, idade e sexo da criança; e a segunda, referente à aplicação de testes. A aplicação das provas motoras teve duração de 30 a 45 minutos, realizadas uma única vez em uma sala devidamente estruturada.

As áreas foram avaliadas por dez tarefas motoras em cada bateria, que se distribuem de forma progressiva de dificuldade, entre as idades de 2 e 11 anos. De acordo com o manual de avaliação motora de Rosa Neto (2015), para crianças com limitações no funcionamento intelectual, nas habilidades adaptativas práticas e sociais, os testes iniciamse pelo nível 2, considerando que a tabela de classificação do desenvolvimento motor segue outros parâmetros. Assim, iniciou-se pelo nível 2, sendo interrompida a avaliação quando não houve êxito na tarefa proposta. O sucesso na realização de cada tarefa determina um ponto na Idade Motora (IM) correspondente e por meio do cálculo das idades motoras em cada área, realiza-se o cálculo de seu Quociente Motor Geral (QMG) e quociente motor específico para cada um dos itens avaliados (QM1, QM2, QM3, QM4, QM5, QM6). 


\section{Análise de dados}

Os resultados foram obtidos por meio do cálculo do quociente motor geral (QMG), que é realizado com base na divisão da idade motora geral (IMG) pela idade cronológica (IC) e multiplicado por cem (QMG = IMG/IC x 100), obtendo uma pontuação que equivale à função motora geral. Com base nessa pontuação os quocientes motores são classificados em muito superior (130 ou mais), superior (120-129), normal alto (110-119), normal médio (90109), normal baixo (80-89), inferior (70-79) e muito inferior (69 ou menos).

A análise estatística foi realizada por meio do programa Microsoft Office Excel, versão 2010. Para verificar a correlação entre a variável Idade Cronológica e Idade Motora Geral utilizou-se a Correlação Linear de Pearson, com nível de significância para as análises de $\mathrm{p}<0,05$.

\section{Resultados}

Participaram do estudo 20 crianças, de ambos os sexos, com diagnóstico de TEA, com idades entre 5 e 11 anos, sendo $14(70 \%)$ do gênero masculino e $6(30 \%)$ do gênero feminino. A tabela 1 apresenta a distribuição amostral segundo a faixa etária, gênero, nível de desenvolvimento motor e escolaridade.

Tabela 1 - Distribuição amostral por idade, sexo, nível de comprometimento e escolaridade, Teresina/PI, Brasil $(n=20)$.

\begin{tabular}{lclll}
\hline $\begin{array}{c}\text { Idade } \\
\text { cronológica }\end{array}$ & Total & Sexo & Classificação & Escolaridade \\
\hline $\begin{array}{c}60 \text { a } 71 \text { meses } \\
\text { (5 anos) }\end{array}$ & 1 & Masculino & Muito inferior & 2 período \\
$\begin{array}{c}72 \text { a } 83 \text { meses } \\
\text { (6 anos) }\end{array}$ & 1 & Masculino & Muito inferior & Não referido \\
$\begin{array}{c}84 \text { a } 95 \text { meses } \\
(7 \text { anos })\end{array}$ & 3 & $F(1), M(2)$ & Muito inferior/inferior & $2^{\circ}$ ano \\
$\begin{array}{c}96 \text { a } 107 \text { meses } \\
(8 \text { anos })\end{array}$ & 8 & $F(3), M(5)$ & Muito inferior & $2^{\circ}, 3^{\circ}$ e $7^{\circ}$ ano \\
$\begin{array}{c}120 \text { a } 131 \text { meses } \\
(10 \text { anos })\end{array}$ & 3 & Masculino & Muito inferior & $2^{\circ}$ ao $4^{\circ}$ ano \\
$\begin{array}{c}132 \text { ou mais } \\
(11 \text { anos })\end{array}$ & 4 & $F(2), M(2)$ & Muito inferior & $4^{\circ}$ ao $6^{\circ}$ ano \\
\hline
\end{tabular}

Legenda: $F=$ feminino, $M=$ masculino. Fonte: Dados da pesquisa

Fonte: Dados da pesquisa. 
Com base na tabela, a faixa etária incluiu crianças de 5, 6, 7, 8, 10 e 11 anos. O nível de desenvolvimento motor apresentado foi inferior e muito inferior. O grau de escolaridade variou do segundo período do ensino infantil ao sétimo ano do ensino fundamental. $O$ desempenho motor não diferiu em relação à escolaridade das crianças avaliadas, podendo ser observado que o desenvolvimento motor classificado como muito inferior, por exemplo, foi verificado em crianças do segundo ao sétimo ano. Assim, é possível identificar que as crianças apresentaram déficits motores significativos, independente da idade ou grau de escolaridade.

Para a análise dos resultados, os dados motores foram tabulados em meses e analisados com base na EDM. Observou-se que $80 \%$ da amostra obteve classificação "muito inferior", com quociente motor menor que 70, demonstrando fator de risco grave para o desenvolvimento motor; $20 \%$ das crianças foram classificadas na categoria "Inferior", apresentando um fator de risco moderado para o desenvolvimento, de acordo com a Tabela 2. Os fatores de risco representam o valor do impacto para o desenvolvimento.

Tabela 2 - Classificação geral do desenvolvimento motor da amostra conforme a EDM, Teresina/PI, Brasil $(n=20)$.

\begin{tabular}{lcccl}
\hline Classificação & Valores & Frequência & $\%$ & Fator De Risco \\
\hline Muito Superior & $>130$ & - & - & Nenhum \\
Superior & $129-120$ & - & - & Nenhum \\
Normal Alto & $119-110$ & - & - & Nenhum \\
Normal Médio & $109-110$ & - & - & Nenhum \\
Normal Baixo & $89-80$ & - & - & Risco Leve \\
Inferior & $79-70$ & 2 & $20 \%$ & Risco Moderado \\
Muito Inferior & $<70$ & 18 & $80 \%$ & Risco Grave \\
\hline
\end{tabular}

Fonte: Dados da pesquisa

A tabela 3 apresenta o resultado da avaliação motora em cada uma das variáveis. Verifica-se que a idade cronológica média foi de 106,7 meses, idade motora geral de 53,4 meses e quociente motor geral de 59,0 meses. Observa-se um atraso motor médio de 49,3 meses, determinado pela diferença entre a idade motora geral e a idade cronológica. Essa diferença não variou em relação ao sexo da criança. Das variáveis avaliadas, observa-se um atraso maior na área de linguagem/organização temporal, apresentando um quociente motor médio de 44,3. 
Tabela 3 - Avaliação do desenvolvimento motor em cada uma das variáveis, Teresina/PI, Brasil.

\begin{tabular}{l|l|l|l}
\hline Variáveis & Média & Mínimo & Máximo \\
\hline Motricidade fina - QM1 & $58,6 \pm 18,1$ & 35 & 93 \\
\hline Motricidade global - QM2 & $58,1 \pm 13,3$ & 33 & 89 \\
\hline Equilíbrio - QM3 & $53,3 \pm 22,9$ & 19 & 123 \\
\hline Esquema corporal - QM4 & $53,9 \pm 16,2$ & 0 & 72 \\
\hline Organização espacial - QM5 & $56,1 \pm 20,0$ & 22 & 93 \\
\hline Organização temporal - QM6 & $44,3 \pm 23,5$ & 0 & 89 \\
\hline
\end{tabular}

Fonte: dados da pesquisa

Todas as crianças apresentaram perfil motor inferior à idade cronológica no momento da avaliação, como demonstrado no gráfico 1. Com relação à lateralidade, 13 sujeitos demonstraram homogeneidade (destro completo); 4 apresentaram lateralizada cruzada, 1 sinistro completo e os demais lateralidade indefinida.

Gráfico 1- Distribuição das médias das variáveis analisadas com idade representa em meses.

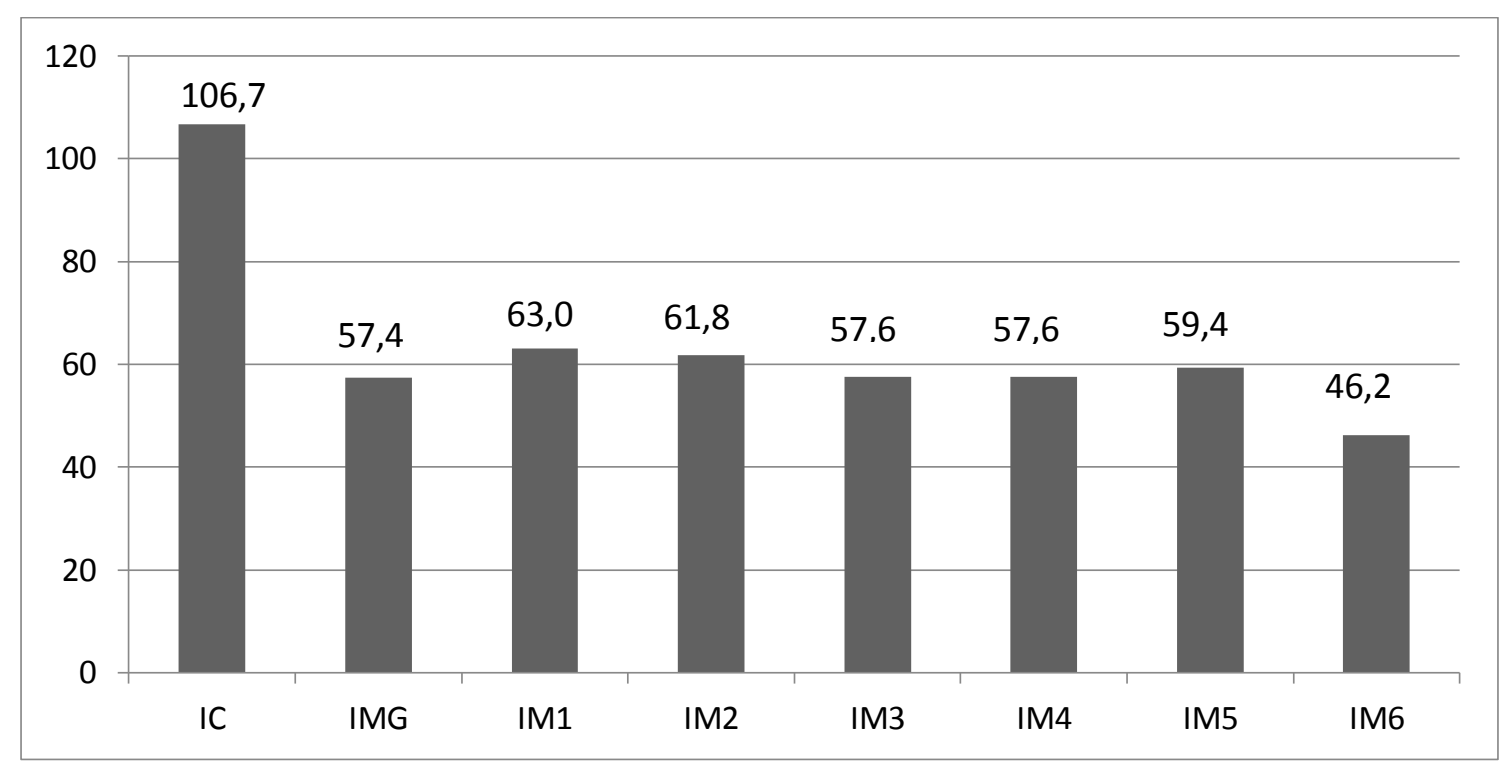

Legenda: IC: idade cronológica, IMG: idade motora geral, IM1: motricidade fina, IM2: motricidade global, IM3: equilíbrio, IM4: esquema corporal, IM5: organização espacial, IM6: organização temporal.

Fonte: Dados da pesquisa.

$\mathrm{Na}$ área da linguagem/organização temporal (IM6) todas as crianças avaliadas apresentaram o pior desempenho motor. Um desempenho inferior também foi observado em motricidade global (IM2) e esquema corporal/rapidez (IM4). Observa-se ainda que em todas as variáveis analisadas houve atrasos motores. $O$ quadro 1 descreve as atividades propostas para a análise motora. 


\section{http://dx.doi.org/10.5902/1984686X33648}

Quadro 1 - Descrição das provas de avaliação motora de cada item da EDM

\begin{tabular}{|c|c|c|c|c|c|c|}
\hline Níveis & $\begin{array}{l}\text { Motricidade } \\
\text { Fina }\end{array}$ & $\begin{array}{l}\text { Motricidade } \\
\text { global }\end{array}$ & Equilíbrio & $\begin{array}{c}\text { Esquema } \\
\text { corporal/Rapidez }\end{array}$ & $\begin{array}{l}\text { Organização } \\
\text { espacial }\end{array}$ & $\begin{array}{c}\text { Linguagem/Organização } \\
\text { temporal }\end{array}$ \\
\hline 2 & $\begin{array}{l}\text { Construção } \\
\text { de uma torre } \\
\text { com cubos }\end{array}$ & $\begin{array}{l}\text { Subir sobre } \\
\text { um banco }\end{array}$ & $\begin{array}{l}\text { Equilíbrio } \\
\text { estático } \\
\text { sobre um } \\
\text { banco }\end{array}$ & $\begin{array}{c}\text { Imitação de } \\
\text { gestos simples } \\
\text { de mãos e braços } \\
\text { (7-12 acertos) }\end{array}$ & $\begin{array}{c}\text { Tabuleiro figuras } \\
\text { geométricas }\end{array}$ & $\begin{array}{l}\text { Linguagem expressiva } \\
\text { (frase de duas palavras) }\end{array}$ \\
\hline 3 & $\begin{array}{l}\text { Construção } \\
\text { de uma ponte } \\
\text { com cubos }\end{array}$ & $\begin{array}{l}\text { Saltar } \\
\text { sobre uma } \\
\text { corda }\end{array}$ & $\begin{array}{l}\text { Equilíbrio } \\
\text { sobre um } \\
\text { joelho }\end{array}$ & $\begin{array}{c}\text { Imitação de } \\
\text { gestos simples } \\
\text { de mãos e braços } \\
\text { (7-12 acertos) }\end{array}$ & $\begin{array}{l}\text { Tabuleiro } \\
\text { invertido }\end{array}$ & $\begin{array}{l}\text { Linguagem expressiva } \\
\text { (frase de seis a sete } \\
\text { sílabas) }\end{array}$ \\
\hline 4 & $\begin{array}{c}\text { Enfiar a linha } \\
\text { na agulha }\end{array}$ & $\begin{array}{l}\text { Saltar } \\
\text { sobre o } \\
\text { mesmo } \\
\text { lugar }\end{array}$ & $\begin{array}{l}\text { Equilíbrio } \\
\text { com o } \\
\text { tronco } \\
\text { flexionado }\end{array}$ & $\begin{array}{c}\text { Imitação de } \\
\text { gestos simples } \\
\text { de mãos e braços } \\
\text { (13-16 acertos) }\end{array}$ & $\begin{array}{l}\text { Prova dos } \\
\text { palitos }\end{array}$ & $\begin{array}{c}\text { Linguagem expressiva } \\
\text { (repetir frases de } 5 \text { a } 6 \\
\text { palavras) }\end{array}$ \\
\hline 5 & Fazer um nó & $\begin{array}{l}\text { Saltar uma } \\
\text { altura de } \\
20 \mathrm{~cm}\end{array}$ & $\begin{array}{c}\text { Equilíbrio } \\
\text { na ponta } \\
\text { dos pés }\end{array}$ & $\begin{array}{c}\text { Imitação de } \\
\text { gestos simples } \\
\text { de mãos e braços } \\
\text { (17-20 acertos) }\end{array}$ & $\begin{array}{l}\text { Jogo de } \\
\text { paciência (dois } \\
\text { triângulos e um } \\
\text { retângulo) }\end{array}$ & $\begin{array}{c}\text { Linguagem expressiva } \\
\text { (repetir frases } \\
\text { de } 7 \text { a } 8 \text { palavras) }\end{array}$ \\
\hline 6 & Labirinto & $\begin{array}{l}\text { Caminhar } \\
\text { em linha } \\
\text { reta }\end{array}$ & $\begin{array}{l}\text { Pé manco } \\
\text { estático }\end{array}$ & $\begin{array}{l}\text { Prova de rapidez } \\
\text { com papel } \\
\text { quadriculado (57- } \\
73 \text { traços) }\end{array}$ & $\begin{array}{l}\text { Conhecimento } \\
\text { sobre si (direita } \\
\text { e esquerda) }\end{array}$ & $\begin{array}{c}\text { Estruturas temporais (6-13 } \\
\text { acertos) }\end{array}$ \\
\hline 7 & $\begin{array}{c}\text { Bolinhas de } \\
\text { papel }\end{array}$ & Pé manco & $\begin{array}{l}\text { Fazer um } \\
\text { quatro }\end{array}$ & $\begin{array}{l}\text { Prova de rapidez } \\
\text { com papel } \\
\text { quadriculado ( } 74- \\
90 \text { traços) }\end{array}$ & $\begin{array}{c}\text { Execução de } \\
\text { movimentos de } \\
\text { direita e } \\
\text { esquerda }\end{array}$ & $\begin{array}{c}\text { Estruturas temporais (14-18 } \\
\text { acertos) }\end{array}$ \\
\hline 8 & $\begin{array}{l}\text { Ponta do } \\
\text { polegar/ } \\
\text { dedos }\end{array}$ & $\begin{array}{l}\text { Saltar uma } \\
\text { altura de } \\
40 \mathrm{~cm}\end{array}$ & $\begin{array}{l}\text { Equilíbrio } \\
\text { de } \\
\text { cócoras }\end{array}$ & $\begin{array}{l}\text { Prova de rapidez } \\
\text { com papel } \\
\text { quadriculado ( } 91 \text { - } \\
99 \text { traços) }\end{array}$ & $\begin{array}{c}\text { Reconhecimento } \\
\text { sobre o outro } \\
\text { (direita e } \\
\text { esquerda) }\end{array}$ & $\begin{array}{c}\text { Estruturas temporais (19-23 } \\
\text { acertos) }\end{array}$ \\
\hline 9 & $\begin{array}{l}\text { Lançamento } \\
\text { com uma bola }\end{array}$ & $\begin{array}{c}\text { Saltar } \\
\text { sobre o ar }\end{array}$ & $\begin{array}{c}\text { Equilíbrio } \\
\text { com o } \\
\text { tronco } \\
\text { flexionado } \\
\text { e ponta } \\
\text { dos pés }\end{array}$ & $\begin{array}{c}\text { Prova de rapidez } \\
\text { com papel } \\
\text { quadriculado } \\
\text { (100-106 traços) }\end{array}$ & $\begin{array}{c}\text { Reprodução } \\
\text { humana } \\
\text { (demonstração e } \\
\text { execução) }\end{array}$ & $\begin{array}{c}\text { Estruturas temporais (24-26 } \\
\text { acertos) }\end{array}$ \\
\hline 10 & $\begin{array}{c}\text { Círculo com } \\
\text { o } \\
\text { polegar }\end{array}$ & $\begin{array}{l}\text { Pé manco } \\
\text { com } \\
\text { retângulo } \\
\text { de madeira }\end{array}$ & $\begin{array}{l}\text { Equilíbrio } \\
\text { na ponta } \\
\text { dos pés } \\
\text { com olhos } \\
\text { fechados }\end{array}$ & $\begin{array}{c}\text { Prova de rapidez } \\
\text { com papel } \\
\text { quadriculado } \\
\text { (107-114 traços) }\end{array}$ & $\begin{array}{c}\text { Boneco } \\
\text { esquemático } \\
\text { (cartões com } \\
\text { figuras e } \\
\text { execução) }\end{array}$ & $\begin{array}{l}\text { Estruturas temporais (27-31 } \\
\text { acertos) }\end{array}$ \\
\hline 11 & $\begin{array}{l}\text { Agarrar uma } \\
\text { bola }\end{array}$ & $\begin{array}{l}\text { Saltar } \\
\text { sobre uma } \\
\text { cadeira }\end{array}$ & $\begin{array}{l}\text { Pé manco } \\
\text { com olhos } \\
\text { fechados }\end{array}$ & $\begin{array}{l}\text { Prova de rapidez } \\
\text { com papel } \\
\text { quadriculado } \\
\text { (115 ou mais) }\end{array}$ & $\begin{array}{l}\text { Posição de três } \\
\text { objetos }\end{array}$ & $\begin{array}{c}\text { Estruturas temporais (32-40 } \\
\text { acertos) }\end{array}$ \\
\hline
\end{tabular}

Fonte: Adaptado de ROSA NETO. p. 46-73 (2002).

A análise de correlação linear de Pearson revelou significância entre IC e IM dos participantes para motricidade fina $(r=0,501)$; motricidade global $(r=0,634)$; equilíbrio $(r=$ 
$0,423)$; e esquema corporal $(r=0,475)$, todos com $p<0,05$. De acordo com o estudo, não houve variação significativa entre as variáveis do estudo e os diferentes graus de escolaridade das crianças, uma vez que os diferentes níveis de escolarização apresentaram as mesmas classificações de desempenho motor.

\section{Discussão}

A aquisição de habilidades motoras geralmente não varia nos primeiros anos de vida, principalmente dos dois aos seis anos, embora o ritmo seja diferente em cada criança. Dessa forma, é possível entender o desenvolvimento motor como um processo de maturação neurológica, somado ao ambiente, às tarefas e ao indivíduo e desenvolvido a partir de uma estimulação aleatória (FÉLIX; ROCHA, 2009).

Com o avançar da idade, mudanças substanciais no comportamento motor ocorrem. Logo, o sistema corporal vai se reorganizando à medida que as proporções corporais mudam, dessa forma influenciando no comportamento e habilidades motoras. Para Gonzaga et al. (2015), a experiência é um importante fator que contribui para o crescimento e maturação. Assim, afirma que em crianças com o espectro autista, para que ocorra a aprendizagem de atos motores, a experiência motora deve ser concedida, como a realização de atividades e jogos lúdicos que estimulem as áreas do desenvolvimento psicomotor.

Silveira et al. (2005) afirma que as crianças passam por diferentes fases em seu desenvolvimento, apresentando evoluções em cada uma delas. No entanto, há uma heterogeneidade nos padrões do desenvolvimento motor que pode estar associada as mais variadas experiências da criança, bem como em seu interesse na realização de atividades. Connoly (2017) afirma que variações nesse padrão podem ser um indicativo de anormalidade e em função disso, testes foram elaborados para avaliar as fases do desenvolvimento.

Para Manoel (2000), algumas etapas podem não ser alcançadas, mas continua a existir uma ordem para que se estabeleçam as mudanças. Todavia, pode-se considerar a aprendizagem motora como um processo constante, considerando as experiências a curto, médio e longo prazo.

Algumas aquisições motoras são características da faixa etária. Assim, habilidades motoras perceptivas, relacionadas à consciência corporal, temporal, de espaço e de direção, são frequentemente confusas na faixa etária pré-escolar. Há também maior 
dificuldade em realizar movimentos bilaterais e algumas habilidades motoras manipulativas (finas), embora necessitem de ajuda, estão desenvolvidas. O controle motor fino ainda não está estabelecido completamente e há desenvolvimento maior do controle motor global, não havendo diferenças significativas entre os gêneros (HAYWOOD, GETCHELL, 2004).

De acordo com Matson et al. (2010), a criança com TEA está sujeita a atrasos nas habilidades motoras, sendo evidente nas primeiras idades da criança, o que corrobora com nossos achados, uma vez que os resultados encontrados demonstraram que as crianças avaliadas encontram-se no nível de desenvolvimento motor inferior e muito inferior, com idades motoras abaixo do nível esperado para a idade cronológica. O mesmo pode ser identificado no estudo de Okuda, Misquiatti e Capellini (2010) realizado com pré-escolares de 5 a 10 anos, evidenciando que atrasos motores são característicos em crianças com o transtorno autístico.

No estudo realizado por Anjos et al. (2017) com 30 crianças de 2 a 11 anos com TEA com o objetivo de traçar o perfil psicomotor das crianças com Transtorno do Espectro Autista por meio da Escala de Desenvolvimento Motor, verificou-se que apresentaram nível de desenvolvimento motor superior, com todas as idades motoras superiores em relação às obtidas nesta pesquisa. No entanto, em ambos os estudos observou-se que a idade motora apresentada foi inferior à idade cronológica.

Sandroni, Ciasca e Rodrigues (2015) realizaram um estudo com o objetivo de avaliar o perfil psicomotor de crianças do ensino infantil com necessidades educativas especiais (NEE). Para avaliação foram utilizados o Inventário Portage Operacionalizado, relativo a socialização, cognição, linguagem e autocuidados e a Escala de Desenvolvimento Motor, para avaliação do perfil psicomotor. Demonstram em seus achados que pré-escolares com TEA apresentaram na avaliação, antes da intervenção psicomotora breve, atrasos no desenvolvimento motor, com todas as idades motoras inferiores às esperadas para a idade cronológica. Os dados obtidos se assemelham aos apresentados nesta pesquisa, uma vez que todas as crianças avaliadas obtiveram padrões motores inferiores ao esperado.

Os resultados normalmente se apresentam de forma não linear em pré-escolares, especificamente em motricidade fina, global, equilíbrio e organização espacial. Silveira et al. (2005), Papst e Marques (2010) afirmam que a não linearidade pode ser explicada em função das habilidades motoras para cada faixa etária serem de forma particular e alguns 
padrões restritos de comportamento estão relacionados às restrições do organismo, do ambiente e da tarefa.

Os achados demonstraram que todas as crianças avaliadas apresentaram atrasos no desenvolvimento motor, considerando os dados da idade motora geral em relação à idade cronológica. Todavia, sabe-se que o desenvolvimento da motricidade fina e global está relacionado com a estimulação, além de considerar os aspectos biológicos e ambientais. Sabe-se ainda que o componente cognitivo apresenta grande influência na aprendizagem motora. Dessa forma, a ausência, bem como a diminuição desses estímulos, pode contribuir para que aspectos do desenvolvimento sejam limitados.

Para a execução de atividades que exigem movimentos finos e precisos, habilidades como atenção e percepção precisam estar desenvolvidas, atributos que estão comprometidos nas crianças com TEA (ROSA NETO et al., 2013). Também se considera a estimulação e o encorajamento em realizar determinada atividade como fatores de desenvolvimento para a motricidade global e espacial (SILVEIRA et al., 2005). Não obstante, os resultados encontrados para a motricidade fina, na atual pesquisa, foram muito inferiores à idade cronológica, tendo em vista o desenvolvimento segundo a EDM.

A não linearidade no desenvolvimento motor para o equilíbrio também pode ser explicada pelas experiências motoras com o ambiente, bem como com a maturação biológica. Verificou-se grande variabilidade do comportamento de acordo com a idade, indicando a particularidade de cada criança. Quanto ao esquema corporal, os resultados foram muito inferiores em relação ao estabelecido pela escala de desenvolvimento. Mastroianni (2006) afirma que são as experiências acumuladas por meio de atividades, brincadeiras que permitem a constituição do esquema corporal e rapidez.

Para Fernandez (2008), o desenvolvimento do equilíbrio estático e lateralidade podem estar prejudicados em crianças com TEA com distúrbios na estruturação do esquema corporal. Os resultados encontrados confirmam esta hipótese, uma vez que se observaram semelhanças no desempenho de tarefas de esquema corporal e equilíbrio.

Vernazza et al (2003) concluíram em seus estudos que os déficits motores relacionados à locomoção e ao equilíbrio em crianças com TEA são consequências da dificuldade em definir uma trajetória de movimento em relação ao alvo. Assim, observou-se que as crianças avaliadas apresentaram desvios significativos nesses elementos, confirmando essa hipótese. 
Desvios significativos nos quocientes motores da motricidade fina e global foram encontrados em pré-escolares com TEA quando comparados aos padrões normais de desenvolvimento nos estudos de Provost et al. (2007), concluindo, assim, que os quocientes motores de crianças com TEA não diferem apenas daquelas com atraso no desenvolvimento neuropsicomotor.

As noções temporais e espaciais estão intimamente ligadas à percepção corporal, sendo base para o desenvolvimento motor, cognitivo e social. Dessa forma, para que estejam desenvolvidas é necessária uma integração com a percepção corporal (FERNANDEZ, 2008). O presente estudo confirma essa teoria, uma vez que os maiores atrasos foram encontrados em esquema corporal e organização temporal e linguagem.

Para Crippa et al. (2003), é importante que a criança consiga estabelecer uma similaridade entre a idade motora e a idade cronológica, tendo em vista que atrasos nessa relação podem ser considerados como fatores limitantes na realização de suas atividades, criando assim a necessidade de correção destas possíveis restrições.

\section{Conclusão}

Após o estudo realizado, foi possível observar que crianças com transtorno do espectro autista (TEA) apresentam idade motora geral inferior à idade cronológica. Nas áreas de motricidade fina, motricidade global, equilíbrio, esquema corporal, organização espacial e temporal e lateralidade obtiveram níveis insatisfatórios, com consequentes padrões motores inferiores do desenvolvimento.

Dessa forma, ressalta-se a importância em avaliar atrasos do desenvolvimento motor em crianças com TEA, identificando precocemente possíveis distúrbios, a fim de prevenir limitações futuras e iniciar intervenções o mais cedo possível.

Em razão da ausência de informações quanto ao diagnóstico, houve dificuldade em definir os diferentes graus do autismo no estudo, pois em grande parte da amostra não houve essa especificação. Assim, trabalhos de natureza interdisciplinar podem ser relevantes para a disseminação de evidências acerca do tema.

Além disso, faz-se necessário um maior embasamento da prática fisioterapêutica na intervenção com o paciente autista. Por isso, discussões dessa temática são importantes para a formação de profissionais qualificados, podendo contribuir para o aumento de evidências e para o desenvolvimento de novas linhas de pesquisa, cada vez mais 
auxiliando para a melhor evolução dessas crianças e esclarecendo aspectos sobre o autismo.

\section{Referências}

AMERICAN PSYCHIATRIC ASSOCIATION. Manual Diagnóstico e Estatístico de Transtornos Mentais DSM-4. 4. ed. Porto Alegre: Artmed, 2014.

ANJOS, Clarissa Cotrim. et al. Perfil Psicomotor de Crianças com Transtorno do Espectro Autista em Maceió/AL. Revista Portal: Saúde e Sociedade. v.2, n.2, p.395410, 2017.

BRASIL. Ministério da Saúde. Secretaria de Atenção à Saúde. Departamento de Ações Programáticas Estratégicas. Diretrizes de Atenção à Reabilitação da Pessoa com Transtorno do Espectro Autista (TEA). Brasília: Ministério da Saúde, p. 86, 2014.

CANUT, Ana Carolina Andrade. et al. Diagnóstico precoce do autismo: relato de caso. Revista de Medicina e Saúde de Brasília, v. 3, n.1, p. 31-37, 2014.

CONNOLY, Kevin. Desenvolvimento motor: passado, presente e futuro. Revista Paulista de Educação Física. São Paulo, p. 6-15, 2017.

CRIPPA, Liamara Regina. et al. Avaliação motora de pré-escolares que praticam atividades recreativas. Revista da Educação Física / UEM. v. 14, n. 2, p. 13-20, 2003.

FÉLIX, Patrícia dos Santos.; ROCHA, Letícia. Ecard. Avaliação motora de préescolares com idade entre 5 e 6 anos que praticam atividades recreativas. Perspectivas online. v.3, n.10, p. 135-137, 2009.

FERNANDEZ, Fabiana S. O corpo no autismo. Revista de Psicologia da Vetor. v.9 n.1, p. 109-14, 2008.

GOMES, Paulyane, T. et al. Autism in Brazil: a systematic review of family challenges and coping strategies. J. Pediatr. v. 91, p. 111-21. Rio de Janeiro, 2015.

GONZAGA, Caroline Nunes. et al. Detecção e intervenção psicomotora em crianças com transtorno do espectro autista. Colloq Vitae, v. 7, n. 3, p. 71-79, 2015.

HAYWOOD, Kathleen. M.; GETCHELL, Nancy. Desenvolvimento motor ao longo da vida. Porto Alegre: Artmed. p.17-33, 2004.

LARSON, Jennifer. C. Gidley. et al. Acquisition of internal models of motor tasks inchildren with autism. Brain, November; v. 11, n. 13, p. 2894-2903, 2008.

MACNEIL, L. K, Mostofsky, Stewart, H. Specificity of dyspraxia in children with autism. Neuropsychology. v. 26, n. 2, p. 167-71, 2012. 
MAGALHÃES, Livia. C.; NASCIMENTO, Viviane. C. S.; REZENDE, Márcia. B. Avaliação da coordenação e destreza motora: etapas de criação e perspectivas de validação. Revista Brasileira de Terapia Ocupacional. São Paulo, v. 15, n. 1, p. 1725, jan./abr., 2004.

MANOEL, Edison de Jesus. Desenvolvimento Motor: padrões em mudança, complexidade crescente. Revista Paulista de Educação Física. n. 3, p. 35-54. São Paulo, 2000.

MATSON, Johnny L. et al. Motor skill abilities in toddlers with autistic disorder, pervasive developmental disorder-not otherwise specified and atypical development.

Res Autism Spect Dis. n. 4. p. 444-49, 2010.

MASTROIANNI, Edelvira de Castro Quintanilha. et al. Abcd no Lar - Aprender, Brincar, Crescer e Desenvolver no Laboratório de Atividades Lúdico - Recreativas. Ed. Unesp. p.557-67. São Paulo, 2006.

MIERES, Ana C. et al. Autism Spectrum Disorder: Anemerging opportunity for physical therapy. Pediatric physical therapy, v. 24, n.1, p. 31-37, 2012.

MOSTOFSKY, Stewart. et al. Developmental dyspraxia is not limited to imitation in children with autism spectrum disorders. Journal of the International Neuropsychological Society, v.12, n.3, p. 314-326, 2006.

NIKOLOV, Roumen.; JONKER, Jacob.; SCAHILL, Lawrence. Autismo: tratamentos psicofarmacológicos e áreas de interesse para desenvolvimentos futuros. Revista Brasileira de Psiquiatria. v. 28, n. 1, p.39-46, 2006.

NITRINI, Ricardo.; BACHESCHI, Luiz Alberto. A neurologia que todo médico deve saber. 2. ed. p.395-397. São Paulo: Atheneu, 2008.

OKUDA, Paola Matiko; MISQUIATTI, Andrea Regina Nunes; CAPELLINI, Simone Aparecida. Caracterização do perfil motor de escolares com transtorno autístico. Revista Educação Especial. v. 23, n. 38, p. 443-454, 2010.

OLIVEIRA, Guiomar. Autismo: diagnóstico e orientação Parte I - Vigilância, rastreio e orientação nos cuidados primários de saúde . Acta Pediatr. v. 40, n. 6, p. 278-87, 2009.

OLIVEIRA, Tania Ribeiro. A intervenção precoce no autismo e trissomia 21: orientações para boas práticas de intervenção. 2010. 68f. Dissertação (Mestrado em Psicologia da Educação). Faculdade de Psicologia e de Ciências da Educação. Coimbra, 2010.

ONZI, Franciele Zanella; GOMES, Roberta Figueiredo. Transtorno do Espectro Autista: A importância do diagnóstico e reabilitação. Caderno pedagógico, Lajeado, v. 12, n. 3, p. 188-199, 2015. 
PAPST, Josiane Medina; MARQUES, Inara. Avaliação do desenvolvimento motor de crianças com dificuldades de aprendizagem. Revista Brasileira de Cineantropometria e Desempenho Humano. v. 12, n. 1, p. 37-41, 2010.

PROVOST, Beth.; HEIMERL, Sandra; LOPEZ, Brian A comparison of motor delays in Young children: Autism Spectrun Disorder, developmental delay, and developmental concerns. J Autism Dev Disord; v. 37, p. 321-28, 2007.

PULZI, Wagner; RODRIGUES, Graciele Massoli. Transtorno do Desenvolvimento da Coordenação: uma Revisão de Literatura. Revista Brasileira de Educação Especial, v. 21 , n. 3, p. 433-444, set. 2015.

ROSA NETO, F. et al. Efeitos da intervenção motora em uma criança com transtorno do espectro do autismo. Temas sobre Desenvolvimento. v. 19, n.105, p. 110-4, 2013.

ROSA NETO, Francisco. et al. A importância da avaliação motora em escolares: análise da confiabilidade da escala de desenvolvimento motor. Revista Brasileira de Cineantropometria e Desempenho Humano. v. 12, n. 6, p. 422-427, 2010.

ROSA NETO, Francisco. Manual de Avaliação Motora. Porto Alegre: Artmed, 2002.

ROSA NETO, Francisco. Manual de Avaliação Motora. 3 ed. Florianópolis: DIOESC, 2015.

SACREY, Lori-ann. et al. Reaching and grasping in autism spectrum disorder: a review of recent literature. In Neurology. p. Canadá, 2014.

SANDRONI, Giuseppina Antonia; CIASCA, Sylvia Maria; RODRIGUES, Sonia das Dores. Avaliação da evolução do perfil motor de pré-escolares com necessidades educativas especiais após intervenção psicomotora breve. Rev. Psicopedagogia. v. 32, n. 97: 4-13. São Paulo, 2015.

SILVEIRA, Caroline Rodrigues Alves. et al. Avaliação motora de pré-escolares: relações entre idade motora e idade cronológica. Revista Digital. n. 83, p. 1-6. Buenos Aires, 2005.

SEGURA, Dora de Castro Agulhon; NASCIMENTO, Fabiano Carlos do; KLEIN, Daniele. Estudo do conhecimento clínico dos profissionais da fisioterapia no tratamento de crianças autistas. Arq. Ciênc. Saúde UNIPAR, v. 15, n. 2, p. 159-165. Umuarama, 2011.

VERNAZZA, Sylvie Martin. et al. Goal Directed Locmotion and Balance Control in Autistic Children. J Autism Dev Disord. v. 35, p. 91-102, 2003. 
WILLRICH, Aline; AZEVEDO, Camila Cavalcanti Fatturi; FERNANDES, Juliana Oppitz. O desenvolvimento motor na infância: influência dos fatores de risco e programas de intervenção. Revista de Neurociências. v.17, n.1, p. 51-56, 2009.

\section{Correspondência}

Bruna Marques Teixeira - Universidade Estadual do Piauí (UESPI). Rua Olavo Bilac, 2335 - Centro (Sul), Teresina. CEP: 64001-280. Teresina, Piauí, Brasil.

\section{(c) (i) (8)}

This work is licensed under a Creative Commons Attribution-NonCommercial 4.0 International (CC BY-NC 4.0) 